\title{
Non-invasive cortical stimulation improves post-stroke attention decline
}

\author{
Eun Kyoung Kang ${ }^{\mathrm{a}}$, Min Jae Baek ${ }^{\mathrm{b}}$, SangYun Kim ${ }^{\mathrm{b}, \mathrm{c}}$ and Nam-Jong Paik ${ }^{\mathrm{a}, \mathrm{b}, \mathrm{d}, *}$ \\ ${ }^{a}$ Department of Rehabilitation Medicine, Seoul National University Bundang Hospital, Seongnam, South Korea \\ ${ }^{\mathrm{b}}$ Clinical Neuroscience Center, Seoul National University Bundang Hospital, Seongnam, South Korea \\ ${ }^{\mathrm{c}}$ Department of Neurology, Seoul National University College of Medicine, Seoul, South Korea \\ ${ }^{\mathrm{d}}$ Department of Rehabilitation Medicine, Seoul National University College of Medicine, Seoul, South Korea
}

\begin{abstract}
Purpose: Attention decline after stroke is common and hampers the rehabilitation process, and non-invasive transcranial direct current stimulation (tDCS) has the potential to elicit behavioral changes by modulating cortical excitability. The authors tested the hypothesis that a single session of non-invasive cortical stimulation with excitatory anodal tDCS applied to the left dorsolateral prefrontal cortex (DLPFC) can improve attention in stroke patients.

Methods: Ten patients with post-stroke cognitive decline (MMSE $\leqslant 25)$ and 10 age-matched healthy controls participated in this double blind, sham-controlled, crossover study involving the administration of real (2 mA for $20 \mathrm{~min})$ or sham stimulation ( $2 \mathrm{~mA}$ for $1 \mathrm{~min}$ ) to the left DLPFC. Attention was measured using a computerized Go/No-Go test before and after intervention. Improvements in accuracy and speed after stimulation relative to baseline were compared for real and sham stimulations.

Results: In healthy controls, no significant improvement in Go/No-Go test was observed after either real or sham stimulation. However, in stroke patients, tDCS led to a significant improvement in response accuracy at 1 hour post-stimulation relative to baseline, and this improvement was maintained until 3 hours post-stimulation $(P<0.05)$, whereas sham stimulation did not lead to a significant improvement in response accuracy $(P>0.05)$. Changes in reaction times were comparable for the two stimulations $(P>0.05)$.

Conclusion: Non invasive anodal tDCS applied to the left DLPFC was found to improve attention versus sham stimulation in stroke patients, which suggests that non-invasive cortical intervention could potentially be used during rehabilitative training to improve attention.
\end{abstract}

Keywords: Attention, cognition, stroke, cortical stimulation, tDCS

\section{Introduction}

Attention decline after stroke is common and hampers the rehabilitation process, reduces physical function recovery, and increases dependence on others during the activities of daily life (McDowd et al., 2003). Furthermore, this problem persists despite pharmacotherapeutic and cognitive rehabilitative ef-

*Corresponding author: Nam-Jong Paik, MD, PhD, Department of Rehabilitation Medicine, Seoul National University College of Medicine, Seoul National University Bundang Hospital 300 Gumidong, Bundang-gu, Seongnam-si, Gyeonggi-do, 463-707, South Korea. Tel.: +82 31787 7731; Fax: +82 31712 3913; E-mail: njpaik@ snu.ac.kr. forts (Coulthard et al., 2006). Accordingly, more effective interventions are required to improve attention after stroke.

Recently, transcranial direct current stimulation (tD$\mathrm{CS}$ ), a form of non-invasive cortical stimulation, which modulates cortical excitability at stimulated sites, has been investigated to determine whether it can facilitate motor (Hummel et al., 2005) and language (Monti et al., 2008) recovery in stroke patients. Furthermore, tDCS has been reported to enhance working memory and attention by stimulating the dorsolateral prefrontal cortex (DLPFC) in healthy subjects (Fregni et al., 2005), and in patients with Parkinson's disease (Boggio et al., 2006). 


\begin{tabular}{|c|c|c|c|c|c|c|}
\hline \multicolumn{7}{|c|}{$\begin{array}{c}\text { Table } 1 \\
\text { Patient data }\end{array}$} \\
\hline Patient' No. & Sex & $\begin{array}{c}\text { Age } \\
\text { (years) }\end{array}$ & Type of stroke (Brain lesion) & $\begin{array}{c}\text { Days } \\
\text { after onset }\end{array}$ & MMSE & 1st Session \\
\hline 1 & $\mathrm{~F}$ & 75 & Infarction (Right corona radiata) & 23 & 19 & Sham \\
\hline 2 & $\mathrm{~F}$ & 66 & Infarction (Both PVWM, right parietal lobe) & 3875 & 20 & Real \\
\hline 3 & M & 48 & Infarction (Right fronto-parietal lobes, basal ganglia) & 1234 & 21 & Sham \\
\hline 4 & M & 74 & Infarction (Left fronto-parieto-temporal lobes) & 47 & 22 & Real \\
\hline 5 & M & 67 & Infarction (Both PVWM, left pons) & 26 & 25 & Sham \\
\hline 6 & M & 71 & Hemorrhage (Right thalamus) & 77 & 19 & Real \\
\hline 7 & $\mathrm{~F}$ & 84 & Infarction (Right corona radiata) & 27 & 18 & Sham \\
\hline 8 & M & 76 & Hemorrhage (Right basal ganglia) & 50 & 23 & Real \\
\hline 9 & M & 69 & $\begin{array}{l}\text { Infarction (Right fronto-occipital lobes, basal ganglia, } \\
\text { corona radiata) }\end{array}$ & 34 & 24 & Sham \\
\hline 10 & $\mathrm{~F}$ & 69 & Hemorrhage (Right basal ganglia) & 48 & 18 & Real \\
\hline Mean $\pm \mathrm{SE}$ & & $69.9 \pm 3.0$ & & $544.1 \pm 388.6$ & $20.9 \pm 0.8$ & \\
\hline
\end{tabular}

F: female, M: male, MMSE: mini-mental state examination, PVWM: periventricular white matter.

Therefore, we tested the hypothesis that a single session of non-invasive cortical stimulation, in the form of excitatory anodal tDCS applied to the left DLPFC, improves attention in stroke patients.

\section{Method}

\subsection{Subjects}

Ten patients (four females) aged 48 to 84 years with post-stroke cognitive decline (MMSE $\leqslant 25$ ) and 10 age-matched healthy control subjects participated in this study (Table 1). We excluded patients with a cerebellar or brainstem lesion, and those with a metallic foreign body implant, a pacemaker, an artificial cochlear, a history of seizure, or an unstable medical condition, and those who were unable to perform the outcome tasks. Patients who were taking $\mathrm{Na}+$ or $\mathrm{Ca}++$ channel blockers, such as carbamazepine or NMDA receptor antagonists like dextromethorphan, were also excluded. Medications unassociated with the study taken by patients were maintained throughout the experiment.

The experimental protocol was approved by the Institutional Review Board of Seoul National University Bundang Hospital and written informed consent was obtained from all subjects.

\subsection{Experimental design}

After being familiarized with the experimental setting, each of the 10 patients underwent a counter balanced crossover session of anodal tDCS $(2 \mathrm{~mA}$ for 20 minutes) or sham stimulation ( $2 \mathrm{~mA}$ for 1 minute) separated by an interval of at least 2 days. Session orders were randomized, that is, 5 subjects underwent the sham session first and the other 5 underwent tDCS first.

At tDCS sessions, the current was slowly increased to $2 \mathrm{~mA}$ from the onset of stimulation and applied for $20 \mathrm{~min}$, whereas at sham stimulation sessions the current was slowly increased to $2 \mathrm{~mA}$ over $1 \mathrm{~min}$ and then slowly tapered down to 0 . This procedure has been demonstrated to prevent patients differentiating real and sham stimulation (Gandiga et al., 2006; Hummel et al., 2005).

tDCS (Phoresor ${ }^{\circledR}$ II PM850; IOMED ${ }^{\circledR}$ Inc., Salt Lake City, Utah) or sham stimulation was delivered through two $25 \mathrm{~cm}^{2}$ sponge electrodes with an anode positioned over F3 as defined by the international 1020 EEG system (F3 corresponds to the left DLPFC), and a cathode positioned over the contralateral supraorbital region. An identical experimental method was applied to the 10 healthy control subjects. Both patients and the investigator that carried out the behavioral measurements were unaware of the type of intervention, because tDCS and sham were administered by another investigator who did not participate in the behavioral task or data analysis.

\subsection{Outcome measurements}

Regarding the Go/No-Go test, numbers of correct responses and reaction times at baseline (Pre), immediately after stimulation (Post1), and at 1 hour (Post2), and 3 hours post-stimulation (Post3) were recorded. Go/No-Go test involved the presentation of the figures "1" or "2" randomly 30 times using Superlab pro v.4.0 software (Cedrus Corporation, San Pedro, CA). One of these figures was presented in the center of a computer screen for $3000 \mathrm{~ms}$, and subjects were instructed to press a right-side blue key as quickly as possible with 
Table 2

Mean attention, perception of fatigue, task difficulty, and sleep quality levels during night prior to testing (rated using numeric $0 \sim 10$ rating scales; $0=$ lowest level, $10=$ highest level)

\begin{tabular}{|c|c|c|c|c|c|c|c|}
\hline & \multicolumn{2}{|c|}{$\mathrm{tDCS}$} & \multicolumn{2}{|c|}{ Sham } & \multicolumn{3}{|c|}{ ANOVA $_{R M}$} \\
\hline & Before & After & Before & After & $\begin{array}{c}\text { Intervention } \\
\text { effect } \\
\mathrm{P} \text { value }\end{array}$ & $\begin{array}{c}\text { Time effect } \\
\mathrm{P} \text { value }\end{array}$ & $\begin{array}{c}\text { Intervention } \times \\
\text { time } \\
\mathrm{P} \text { value }\end{array}$ \\
\hline Attention & $5.7 \pm 2.3$ & $4.5 \pm 0.9$ & $4.4 \pm 2.8$ & $4.1 \pm 2.7$ & 0.342 & 0.101 & 0.324 \\
\hline Fatigue & $4.7 \pm 1.9$ & $3.9 \pm 2.0$ & $3.7 \pm 2.7$ & $3.1 \pm 2.8$ & 0.262 & 0.163 & 0.811 \\
\hline Difficulty* & - & $3.0 \pm 1.9$ & - & $4.0 \pm 2.9$ & 0.229 & - & - \\
\hline Sleep quality* & $5.2 \pm 2.8$ & - & $5.1 \pm 2.9$ & - & 0.910 & - & - \\
\hline
\end{tabular}

${ }^{*}$ Group task difficulties and sleep qualities were compared using the paired $t$ test.

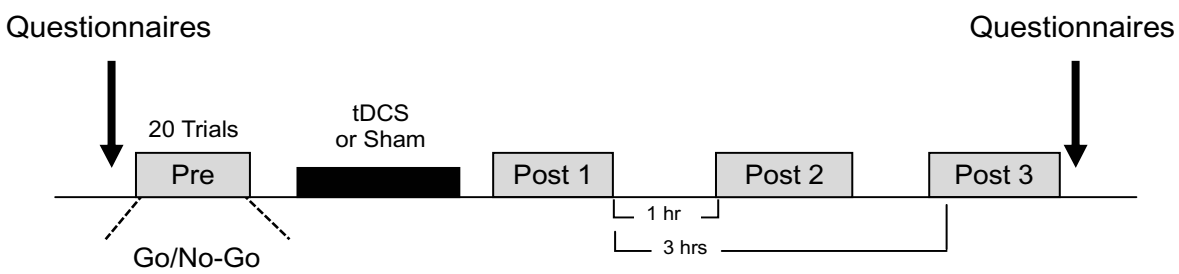

Fig. 1. Experimental design.

the non-paretic hand (the dominant hand for healthy controls) when the figure " 1 " was shown, but not to press any key when the figure " 2 " was shown.

Before and after each session, subjects described their subjective attention levels, perceptions of fatigue, task difficulty, and sleep quality during the previous night using a numeric rating scale (range $0 \sim 10 ; 0=$ lowest, $10=$ highest) (Fig. 1).

Statistical analysis was performed using repeated measures ANOVA (ANOVA $R M$ ) with "INTERVENTION" (tDCS versus Sham) and "TIME" (Pre, Post1, Post2, Post3) as within subject factors. LSD correction for multiple comparisons was performed conditioned on significant $\mathrm{p}$-values $(P<0.05)$. All values are presented as means \pm standard errors (SE).

\section{Results}

\subsection{Effects of non-invasive cortical stimulation on Go/No-Go test results in healthy control subjects}

In the healthy control group (age $=69.3 \pm 2.8$ years; MMSE $=29.2 \pm 0.2$ ), ANOVA $_{\mathrm{RM}}$ showed that INTERVENTION $_{\text {tDCS,Sham, TIME }}$ Pre,Post1,Post2,Post3, or INTERVENTION $_{\text {tDCS,Sham }} \times$ TIME $_{\text {Pre,Post1,Post2, }}$ Post3 had no effect on the number of correct responses or on reaction time as determined by the $\mathrm{Go} / \mathrm{No}-\mathrm{Go}$ test ( $P>0.05$; Fig. 2-A).

\subsection{Effects of non-invasive cortical stimulation on Go/No-Go test results in stroke patients}

In the patient group (age $=69.9 \pm 3.0$ years; MMSE $=20.9 \pm 0.8$ ), numbers of correct responses (control $29.9 \pm 0.1$ versus patients $23.4 \pm 2.1 ; P=0.006)$ was significantly decreased and reaction times (control $682.2 \pm 49.3 \mathrm{~ms}$ versus patients $1267.6 \pm 97.3 \mathrm{~ms}$; $P<0.001)$ lengthened respectively, versus the 10 agematched healthy control group, indicating that attention was lower in the patient group.

ANOVA $_{R M}$ revealed that INTERVENTION ${ }_{\text {tDCS, }}$ Sham, TIME $E_{\text {Before,After }}$, or INTERVENTION tDCS,Sham $_{\text {, }}$ $\times$ TIME $_{\text {Before,After }}$ had no effect on subjective ratings of attention and fatigue $(P>0.05)$. Furthermore, the paired t test found no difference between sham and real intervention sessions in terms of task difficulty or sleep quality during the previous night, indicating that gross psychophysical conditions during tDCS and Sham administration were comparable $(P>0.05$; Table 2$)$.

According to Go/No-Go test results, baseline mean correct response numbers (tDCS $23.4 \pm 2.1$ versus Sham $26.1 \pm 1.9$, paired t-test $P=0.15)$ and reaction times (tDCS $1267.6 \pm 97.3 \mathrm{~ms}$ versus Sham $1097.0 \pm$ $94.2 \mathrm{~ms}, P=0.15)$ were comparable for tDCS and Sham administration.

ANOVA $_{R M}$ revealed a significant INTERVENT$\mathrm{ION}_{\mathrm{tDCS}, \text { Sham }} \times \mathrm{TIME}_{\text {Pre,Post1, Post2, Post3 }}$ effect $[\mathrm{F}$ $=3.11 ; P=0.043$ ], but no significant INTERVENT$\mathrm{ION}_{\mathrm{tDCS}, \text { Sham }}[\mathrm{F}=0.01 ; P=0.92]$ or TIME $\mathrm{Pre}_{\text {Post1, }}$ 


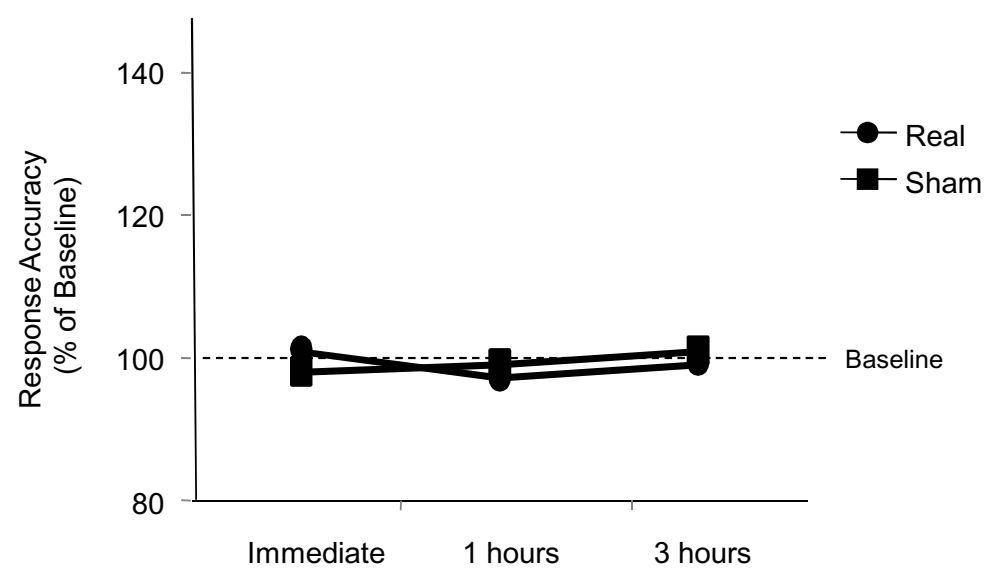

(A)

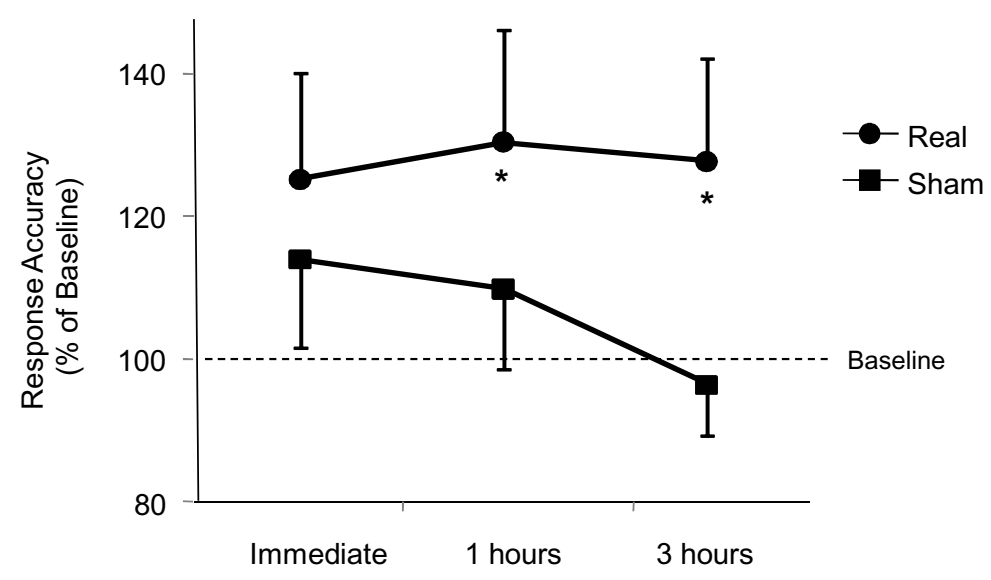

(B)

Fig. 2. Graph showing the effects of anodal tDCS on percentage change in Go/No-Go test accuracy relative to baseline (dotted line, 100\%). (A) No improvement was observed in healthy controls after either tDCS or sham stimulation. (B) Note that tDCS (asterisk) but not Sham resulted in a response accuracy improvement relative to baseline at 1 hour and 3 hours post-stimulation in stroke patients. Values represent means \pm SE ${ }^{*} P<0.05$.

Post2,Post3 $[\mathrm{F}=2.18 ; P=0.11]$ effect on numbers of correct responses. Post hoc testing showed that tDCS induced to a significant improvement in response accuracy at 1 hour post-stimulation relative to baseline (from Pre $23.4 \pm 2.1$ to Post2 27.6 $\pm 1.0 ; P=0.024$; Fig. 2-B), and this improvement was maintained until 3 hours post-stimulation (Post3 27.2 $\pm 0.7 ; P=0.041$; Fig. 2-B, asterisk), whereas sham stimulation did not lead to significant improvement in response accuracy (from Pre $26.1 \pm 1.9$ to Post2 $26.8 \pm 0.8 ; P=0.702$ and to Post3 $24.1 \pm 1.4 ; P=0.276$; Fig. 2-B).

However, ANOVA $_{\mathrm{RM}}$ revealed no significant effect of INTERVENTION tDCS, Sham $\left._{\text {IF }}=0.01 ; P=0.941\right]$,
TIME $_{\text {Pre,Post1,Post2,Post3 }}[\mathrm{F}=0.78 ; P=0.514]$ or INTERVENTION $_{\mathrm{tDCS}, \text { Sham }} \times$ TIME $_{\text {Pre,Post1,Post2, }}$ Post3 $[\mathrm{F}=1.36 ; P=0.28]$ on reaction time.

Regarding the effect of order of stimulation (tDCS first versus sham first), ANOVA $_{R M}$ showed no significant effect of ORDER $\mathrm{tDCS}_{\text {, Sham }}[\mathrm{F}=$ $0.50 ; P=0.497], \quad \mathrm{TIME}_{\text {Pre,Post1,Post2, Post3 }} \quad[\mathrm{F}$ $=2.18 ; \quad P=0.113]$, or ORDER tDCS,Sham $\times$ TIME $_{\text {Pre,Post1,Post2,Post3 }}[\mathrm{F}=0.70 ; P=0.563]$ on numbers of correct responses, and no significant effect of ORDER DCS,Sham $[\mathrm{F}=1.65 ; P=0.231]$, TIME $_{\text {Pre,Post1,Post2,Post3 }}[\mathrm{F}=0.783 ; P=0.514]$, or ORDER $_{\text {tDCS,Sham }} \times$ TIME $_{\text {Pre,Post1,Post2,Post3 }}[\mathrm{F}=$ 
1.204; $P=0.327$ ] on reaction times, indicating that improvements in numbers of correct responses after tDCS session were not caused by order of stimulation.

\section{Discussion}

The main finding of this double blind, sham controlled, cross-over study was that a single session of anodal tDCS applied to the left DLPFC improved attention versus sham stimulation in stroke patients.

Cognitive impairments, such as, memory, orientation, and attention deficit frequently occur after stroke, though sometimes, attentional deficit is the only cognitive impairment apparent (Tatemichi et al., 1994). Sustaining attention is prerequisite of motor relearning (McNevin et al., 2000), which is essentially the mechanism of functional recovery after stroke (Hikosaka et al., 2002). The traditional neurocognitive approaches (Coulthard et al., 2006) used to improve attention in stroke patients often result in incomplete resolution, and thus, more effective adjuvant strategies that enhance the effects of neurocognitive training are needed.

tDCS can non-invasively modulate the excitability of targeted brain regions, e.g., anodal stimulation increases, whereas cathodal stimulation decreases cortical excitability (Nitsche and Paulus, 2000). Furthermore, this technique is easily administered in parallel with cognitive rehabilitative training (Gandiga et al., 2006). Recently, tDCS was reported to improve motor (Hummel et al., 2005) and language function (Monti et al., 2008) in stroke patients, and in the cognitive domain, it has been reported that a single session of anodal tDCS applied to the left DLPFC improves working memory task results in healthy subjects (Fregni et al., 2005) and in idiopathic Parkinson's disease (Boggio et al., 2006).

Based on these reports, we hypothesized that a single session of anodal tDCS applied to the left DLPFC would improve attention in stroke patients. We chose the Go/No-Go test because it is known to provide sensitive measures of attention with high test-retest reliability (Aranda et al., 2001).

The DLPFC plays a crucial role during working memory and attention tasks (D'Esposito et al., 1998). It receives somatosensory and visual input information from the parietal heteromodal association cortex regarding peripheral vision, motion, spatial orientation, and tactile sensations from the trunk and extremities, and projects to subcortical monoaminergic and cholinergic sources (Devinsky and D'Esposito, 2004). Accordingly, the DLPFC influences global behaviors, such as, attention and arousal, and any lesion disrupting this network is likely result in an attention or arousal deficit.

In the present study, we found that the application of tDCS to left DLPFC improved Go/No-Go test accuracy in stroke patients, although it did not lead to statistically significant acute (Post1 in the present study) improvements. We speculate that this lack of an acute improvement, which has been found in other studies (Kuo et al., 2008; Nitsche et al., 2003) was due to a limited sample size, the adoption of different behavioral outcome measures, the different natures of populations studied, or to the application of tDCS before the task rather than during the task.

It is notable that comparable subjective ratings of attention levels, perceptions of fatigue, task difficulties, and sleep qualities during previous nights (as determined using numeric rating scales between sessions), and no changes in reaction times argues against the notion that the observed maintenance of accuracy was the result of a non specific effect of tDCS.

It should be noted that we did not monitor mood in this study, and because a depressed mood is common after stroke and might interfere with performance, we cannot rule out the possibility that indirect effects of tDCS reduced this depression, and thus, improved attention during the behavioral task. Furthermore, no extracephalic electrode montage was used in the present study (Priori et al., 2008), and therefore, we also cannot exclude the possibility that the observed beneficial effects were due to placing the cathode on skin overlying the right supraorbital region, which corresponds to the right prefrontal cortex.

In conclusion, noninvasive anodal tDCS applied to left the DLPFC was found to improve attention as compared with sham stimulation, which suggests that tDCS could be used during cognitive or motor rehabilitative training to improve attention.

\section{Acknowledgement}

This research was supported by a grant from Seoul National University College of Medicine (Grant No. 800-20060236) to N.J. Paik, and by a grant from the Korean Geriatric Society to E.K. Kang.

\section{References}

Aranda, D., Bartsch, A. J., Herrmann, M. J., Eisenack, S., Morinigo, J. C., \& Fallgatter, A. J. (2001). [Reliability of electrophysiological measurements of motor control]. Rev Neurol, 32(1), 10-14. 
Boggio, P. S., Ferrucci, R., Rigonatti, S. P., Covre, P., Nitsche, M., Pascual-Leone, A., et al. (2006). Effects of transcranial direct current stimulation on working memory in patients with Parkinson's disease. J Neurol Sci, 249(1), 31-38.

Coulthard, E., Singh-Curry, V., \& Husain, M. (2006). Treatment of attention deficits in neurological disorders. Curr Opin Neurol, 19(6), 613-618.

D’Esposito, M., Aguirre, G. K., Zarahn, E., Ballard, D., Shin, R. K., \& Lease, J. (1998). Functional MRI studies of spatial and nonspatial working memory. Brain Res Cogn Brain Res, 7(1), $1-13$

Devinsky, O., \& D'Esposito, M. (2004). Neurology of cognitive and behavioral disorders. Oxford ; New York: Oxford University Press.

Fregni, F., Boggio, P. S., Nitsche, M., Bermpohl, F., Antal, A., Feredoes, E., et al. (2005). Anodal transcranial direct current stimulation of prefrontal cortex enhances working memory. Exp Brain Res, 166(1), 23-30.

Gandiga, P. C., Hummel, F. C., \& Cohen, L. G. (2006). Transcranial DC stimulation (tDCS): a tool for double-blind shamcontrolled clinical studies in brain stimulation. Clin Neurophysiol, 117(4), 845-850.

Hikosaka, O., Nakamura, K., Sakai, K., \& Nakahara, H. (2002). Central mechanisms of motor skill learning. Curr Opin Neurobiol, 12(2), 217-222.

Hummel, F., Celnik, P., Giraux, P., Floel, A., Wu, W. H., Gerloff, C., et al. (2005). Effects of non-invasive cortical stimulation on skilled motor function in chronic stroke. Brain, 128( $\mathrm{Pt} 3)$, 490-499.

Kuo, M. F., Unger, M., Liebetanz, D., Lang, N., Tergau, F., Paulus, W., et al. (2008). Limited impact of homeostatic plasticity on motor learning in humans. Neuropsychologia, 46(8), 2122 2128.

McDowd, J. M., Filion, D. L., Pohl, P. S., Richards, L. G., \& Stiers, W. (2003). Attentional abilities and functional outcomes following stroke. J Gerontol B Psychol Sci Soc Sci, 58(1), P4553.

McNevin, N. H., Wulf, G., \& Carlson, C. (2000). Effects of attentional focus, self-control, and dyad training on motor learning: implications for physical rehabilitation. Phys Ther, 80(4), 373-385.

Monti, A., Cogiamanian, F., Marceglia, S., Ferrucci, R., Mameli, F., Mrakic-Sposta, S., et al. (2008). Improved naming after transcranial direct current stimulation in aphasia. J Neurol Neurosurg Psychiatry, 79(4), 451-453.

Nitsche, M. A., \& Paulus, W. (2000). Excitability changes induced in the human motor cortex by weak transcranial direct current stimulation. J Physiol, 527 Pt 3, 633-639.

Nitsche, M. A., Schauenburg, A., Lang, N., Liebetanz, D., Exner, C., Paulus, W., et al. (2003). Facilitation of implicit motor learning by weak transcranial direct current stimulation of the primary motor cortex in the human. J Cogn Neurosci, 15(4), $619-626$.

Priori, A., Mameli, F., Cogiamanian, F., Marceglia, S., Tiriticco, M., Mrakic-Sposta, S., et al. (2008). Lie-specific involvement of dorsolateral prefrontal cortex in deception. Cereb Cortex, 18(2), 451-455.

Tatemichi, T. K., Desmond, D. W., Stern, Y., Paik, M., Sano, M., \& Bagiella, E. (1994). Cognitive impairment after stroke: frequency, patterns, and relationship to functional abilities. $J$ Neurol Neurosurg Psychiatry, 57(2), 202-207. 